\title{
Evaluating the Impact of a Virtual Reality Application in Raising Awareness Toward the Destruction of Cultural Heritage Sites
}

\author{
Christos Hadjipanayi ${ }^{1}$, Eleni Demitriadou ${ }^{1}$, Haris Frangou ${ }^{1}$, Maria Papageorgiou ${ }^{1}$, \\ Christina Zavlanou ${ }^{2}$, Andreas Lanitis ${ }^{2,3} 3$ [0000-0001-6841-8065] \\ ${ }^{1}$ Dept. of Multimedia and Graphic Arts, Cyprus University of Technology, Limassol, Cyprus. \\ ${ }^{2}$ Visual Media Computing Research Lab, Department of Multimedia and Graphic Arts, Cyprus \\ University of Technology, Limassol, Cyprus. \\ ${ }^{3}$ Research Centre on Interactive Media Smart Systems and Emerging Technologies, Cyprus.
}

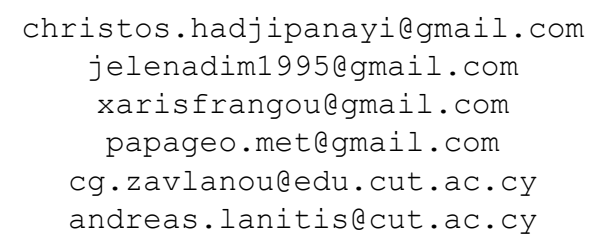

\begin{abstract}
The aim of our work is to investigate the applicability of Virtual Reality (VR) in raising awareness of users in relation to the destruction of important monuments. The proposed methodology involves the exposure of users to three virtual environments displaying the original state of a monument, the current state and the predicted future state of the same monument in the case that the monument is not maintained. The exposure to the three states of the same building allows the user to experience the "glorious days" of a monument and compare them to the current and future states in an attempt to realize the level of destruction that could occur to the building if the monument is not maintained properly. As part of a pilot case study, a number of volunteers were asked to navigate in virtual environments depicting the three chronological states of a landmark building. Preliminary results indicate a significant increase of the intensity of negative emotions of the users, indicating the applicability of VR in alerting the society toward the destruction of important monuments.
\end{abstract}

Keywords: Virtual Reality, Cultural Heritage, Visualization Techniques

\section{Introduction}

Virtual Reality (VR) is defined as "an interactive three-dimensional environment that is created on the computer and in which the user can be immersed" [1]. Recent progress of science and technology resulted in affordable and efficient hardware and tools [2] that enables the design and implementation of engaging applications that can 
be used in a variety of different fields, resulting in an increased popularity of VR in different application domains [3]. A key issue to enable efficient interaction of users with a 3D virtual environment is the "presence" and "immersion" [4] of the users, i.e. the illusion of users that they are in the virtual environment, physically and mentally [5]. Immersive applications engage the users emotionally in a similar way like the exposure to real environments [6] maximizing in that way the impact of a VR application.

The aim of our work is to investigate the applicability of VR in raising awareness of users in relation to the destruction of important monuments. The proposed methodology involves the exposure of users to three virtual environments displaying the original state of a monument, the current state and the predicted future state of the same monument in the case that the monument is not maintained. The exposure to the three states of the same building allows the user to experience the "glorious days" of a monument and compare them to the current and future states in an attempt to realize the level of destruction and the size of the potential loss in the case that the monument is not maintained properly. As part of an experimental investigation changes in the emotions of the users are registered to assess the emotional impact of the application.

In the remainder of the paper we present a brief literature review of the use of VR in Cultural Heritage applications, a description of the VR application and the experimental evaluation. In section 5 conclusions and plans for future work are presented.

\section{Related Work}

Virtual reality can be exploited in various areas of human activity. One of these is the design of sites and buildings that are hardly reachable by visitors either due to distance or area configuration [7]. For example, Behr et al. [8] simulated the cathedral of Siena, Italy. The virtual environment, including a virtual avatar-style guide, dressed in traditional costume, allows users to explore the site and draw important historical information about the temple and its architecture.

Virtual reality could be also used to rebuild buildings and ruins of cultural heritage, to restore their original state in all details [9], thereby promoting the feeling of change of spaces and buildings over time [8] while enabling users to research what they could previously research only by descriptions [10]. Christofi et al. [7] reconstructed the historic site of Choirokitia, in Cyprus, based on true information, aimed at assessing the learning performance of the users and examining whether their interest in archaeological sites is increasing.

Kontogianni et al. [9] reconstructed the Middle Lodge in the Ancient Agora, dating back to the 2nd century BC, situated northwest of the sacred rock of the Acropolis of Athens. The building today does not exist and visitors can see only its foundations. The implementation of the project relies on a bibliographical survey, reconstruction 
studies, plans, three-dimensional reconstruction of the foundations, as well as considering assumptions of the site's managers working in the Ancient Agora.

Loizides et al. [11] describe an evaluation of user experience in relation to the use of VR in presenting Cultural Heritage sights. According to the findings the use of VR for the presentation of VR is highly appealing for the users and as a result it can cause emotional engagement of the users. However, the most important drawback of this approach was the user nauseousness that may cause discomfort to users. Fortunately, improvements in VR hardware resulted in noticeable decrease of nauseousness symptoms to VR users, improving the applicability of VR to different application domains.

\section{VR Application}

In this section we provide information related to the site used in the case study, along with the details related to the implementation of the VR application.

\subsection{Case Study}

The case study considered in this paper, relates to the abandoned hotel "Berengaria", a landmark building in the Troodos area of Cyprus. The hotel was completed in 1931 and it used to be a point of reference for the whole region [12]. During 1984 the operation of the hotel was suspended and since then the impressive building is following a continuous deterioration that will eventually lead to its complete destruction. The hotel is linked to numerous mysterious stories and myths and as a result it is considered a key site for the local region.

\subsection{Virtual representation of the hotel}

For the needs of the experimental investigation it is required to have three models showing the building while it was in operation, the current state of the hotel and a model showing the prediction of its future appearance. It should be noted that for all three cases there is no need to create a geometrically correct 3D model; a close approximation of the appearance of the building is sufficient for the needs of the experiment. Hence this paper is not focused on techniques and methodologies suitable for photorealistic 3D reconstruction.

The 3D modeling process started with the capture of photographs and measurements of the building. This material formed the basis of generating a 3D model of the facade and part of the interior of the building that can be used for generating the $3 \mathrm{D}$ models corresponding to the three chronological phases under investigation. To generate the 3D model showing the past appearance of the building, an extensive literature search was carried out in order to collect photographic material showing the interior and exterior of the hotel during its "glory" days. Photographic material collected 
on site was used to recreate and texture the 3D model based on the current hotel appearance. Since the hotel was abandoned for several years, the current appearance shows the depressing views of worn out walls, destroyed doors and windows, damages from graffiti and nests for reptiles in the building's interior. Because of erosion due to aging, a fire that broke out in 2001 and vandalisms, it is foreseen that the building is in immediate danger of collapsing, hence the model showing the future state of the building was created by using special effects where photographs from the current state and the 3D models are deformed to show the building almost-demolished.

It should be noted that this work is still in progress. Currently the $3 \mathrm{D}$ model showing the past appearance was generated, but the generation of the complete 3D models showing the current and future appearance is still in progress. For the needs of the preliminary experimental investigation, a panorama of photographic material was used to generate the illusion of a 3D model showing the current and future state of the building.

\subsection{Implementation of the Application}

The main software tools used for the generation of 3D models and the implementation of the application were the Autodesk Maya, Unity 3D and Adobe Photoshop 2018. The necessary soundtrack, that includes the provision of aural information about the site, was recorded through Ice-cream screen recorder and edited via Adobe Premiere. The final application works with an Oculus Rift CV1 head mounted display with touch controllers so that the level of immersion of users in the simulated environment [13] is maximized. The user of the application can navigate the site using the VR touch controllers, in first person view. During the navigation information about the hotel is presented to the users through audio channels.

A total of three scenes were planned in the environment. In the first scene, the user was transported to the "glorious" past by looking at the facade. From the main entrance, the user had the option to be transported from the reception area to the mezzanine through the central staircase, and then to the balcony area. In the second scene, the user was confronted with the tragic current state of the building, by looking at the facade. In the third and last scene, the user could witness nothing but scattered relics of the ever-imposing building. Then, moving into the interior, the user could only see the debris that will remain if the state of the building is not maintained. Screenshots from the three scenes depicting different chorological states of the site are shown in Figure 1. 

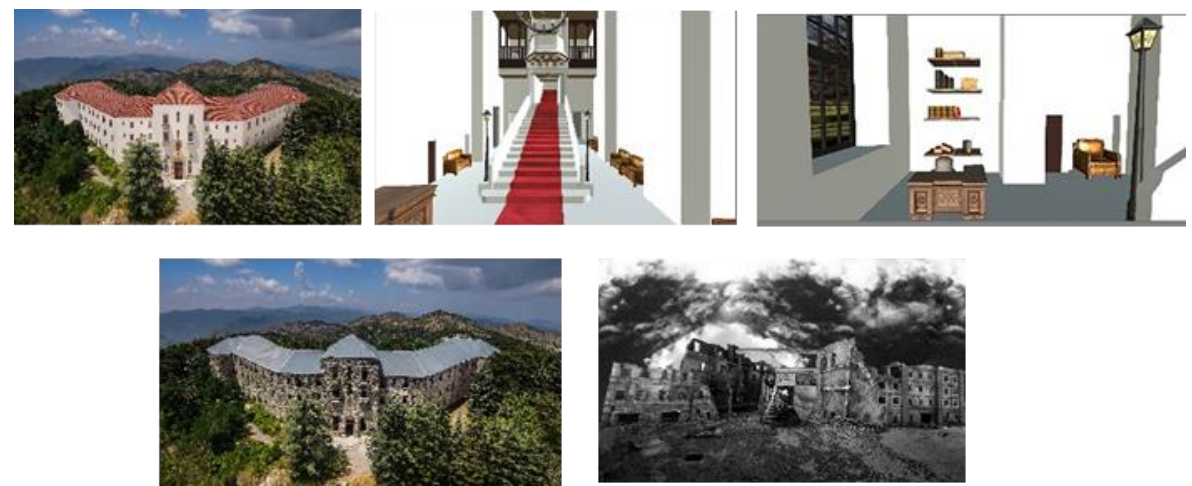

Fig. 1. Facade of the hotel as it was in the past as well as the interior of this hotel (top), current state of the facade (bottom left) and the predicted future appearance (bottom right)

\section{Experimental Evaluation}

An experimental evaluation was carried out to investigate whether the exposure of users to the three VR environments could affect the emotional state of the users in a way that indicates increased awareness and concern related to the need for preventing the destruction of the building. This was accomplished through the examination of changes in the emotional states of the users, before and after the exposure to the VR environments. A description of the experiment and the results are presented hereunder.

\subsection{Participants}

The selection of participants in the survey was made by convenience sampling [14]. Twenty adults, 8 male (40\%), 12 female (60\%), 18-45 years old, with a mean of 26.5 $(\mathrm{SD}=6.243)$ took part in the experiment. Among the sample $17(85 \%)$ participants live in urban areas and $3(15 \%)$ in semi-urban. Twelve $(60 \%)$ of the participants were graduates of a Higher Education Institution, three (15\%) high school graduates, three (15\%) holders of a postgraduate diploma and two (10\%) PhD holders. All survey participants use the internet, with the majority of them, namely $18(90 \%)$ on a daily basis. Most of the participants ( $85 \%$ of the sample), knew what virtual reality is, while half of them had a previous experience with it. Most of them, $13(65 \%)$ also believe that, through the virtual world, they can live and experience similar experiences as in the real / natural world.

\subsection{Data Collection}

Data collection was achieved through two questionnaires [15]. The first questionnaire was completed by the participants at the beginning of the experiment, before they use the application. The first questionnaire had four parts with closed-ended questions. The first part concerns the demographic data of the participants (sex, area of resi- 
dence, age, education) and the second part examines the participants' experience with virtual reality. Specifically, the second part of the questionnaire included questions related to the computer literacy of the participants and their previous experience with virtual reality. In the third part of the questionnaire users had to quote the intensity of their current six basic emotions (Anger, Fear, Disgust, Happiness, Joy, Sadness) on the Likert scale where 1 indicates minimal intensity of the corresponding emotion and 5 indicates maximum intensity of the corresponding emotion. The last part of the questionnaire examines the participants' knowledge of basic facts related to the building such as facts related to the location, opening and closing date, background of its naming and other historical facts.

The second questionnaire was given to the participants after they used the VR application for about five minutes. The questionnaire consisted of four parts. In the first part, there were questions about the user experience in the virtual environment. In the second part feelings of the users were recorded in the same way as in the first questionnaire, in order to detect changes in the intensities of the emotions. The third part of the questionnaire examines the knowledge that users gained through the experience. It should be noted that during the navigation in the VR environment participants were presented with aural information about the site. In this case the aim was to compare the knowledge gained by participants during their overall VR experience. In the last part of the questionnaire, participants had to answer three open questions related to their willingness to help to maintain and restore the building. Participants also stated how they planned to contribute to the maintenance of the site.

\subsection{Data Analysis and Results}

The SPSS statistical packet was used to analyze the quantitative data. Descriptive statistics and the t-test statistical criterion were applied, and paired comparisons were made to determine whether there were statistically significant changes in emotions. The results (see Table 1) indicate that negative emotions of the users were affected. Specifically, there is a strong variation detected and statistical significance $(p<0.05)$ in the feelings of anger, disgust, sadness and fear. On the contrary, positive emotions, such as joy and happiness, did not change significantly. The increase of the negative emotions is a strong indication of the success of the application in raising awareness towards the destruction of the building. Results also indicate a significant improvement in the knowledge gained about the building, and the willingness of the volunteers to contribute to the effort of maintaining/restoring the building. 
Table 1. Comparing the average of emotions before and after users experience with the planned environment (Emotion names in bold indicate the emptions with significant change in intensity)

\begin{tabular}{lllll}
\hline Emotions & $\begin{array}{c}\text { Mean (SD) } \\
\text { Before }\end{array}$ & $\begin{array}{c}\text { Mean (SD) } \\
\text { After }\end{array}$ & T & p-value \\
\hline Anger & $1,60(0,883)$ & $2,10(1,165)$ & 2,127 &, $\mathbf{0 4 7}$ \\
Fear & $1,80(1,005)$ & $2,35(1,137)$ & 3,240 &, 004 \\
Disgust & $1,40(, 995)$ & $2,35(1,268)$ & 4,790 &, 000 \\
Happiness & $3,20(1,105)$ & $3,25(, 967)$ & 170 &, 887 \\
Joy & $3,25(1,020)$ & $3,10(, 912)$ &, 767 &, 453 \\
Sadness & $1,40(, 681)$ & $2,45(1,191)$ & 4,273 &, 000 \\
Surprise & $2,35(1,309)$ & $2,70(1,129)$ & 1,234 &, 232 \\
\hline
\end{tabular}

\section{Conclusions and Future Work}

A preliminary investigation in the use of a VR application for raising awareness towards the destruction of important buildings is described. As part of the experiment, users were exposed to virtual environments showing the past, current and future predicted appearance of a building in the case that the building is not maintained properly. Significant increase of negative emotions of the users was recorded, indicating that such a VR application can have an important impact on users contributing in that way to efforts for preserving Cultural Heritage sites. The use of VR as a means of creating highly immersive and affective applications, can be applied for other Cultural Heritage sites to help raise interest among policy makers and the public. Although other means of raising awareness for the destruction of $\mathrm{CH}$ sites could be employed (i.e. utilization of campaigns through social media), we envisage that VR tools can be more effective for this particular application due to the immersive nature of VR technology.

Based on the promising results of this preliminary investigation we plan to carry on with the 3D modeling phase so that complete 3D models of the three chronological periods are generated. Although so far for the needs of the experiment we did not use photorealistic 3D models of the site, in the future we plan to investigate the impact when geometrically correct models are used in similar applications. We also plan to incorporate damage simulators [16] in order to get better predictions of the foreseen future state of a site. Furthermore, more sophisticated EEG-based methods [17] for recording changes in emotional states will be used so that more accurate results in relation to the impact of the application are recorded. We also plan to further investigate the intensity of awareness raised among the participants in relation to the increase of negative emotions recorded and the content presented in the VR application. We also plan to use the same principle on other sites in order to verify the findings in different scenarios and to contribute to the efforts of preserving Cultural Heritage monuments. 


\section{References}

1. Conn, C., Lanier, J., Minsky, M., Fisher, S., Druin, A.: Virtual environments and interactivity: Windows to the future. In ACM SIGGRAPH Computer Graphics, 23(5), 7-18(1989)

2. Coburn, J. Q., Freeman, I., Salmon, J. L.: A review of the capabilities of current low-cost virtual reality technology and its potential to enhance the design process. Journal of Computing and Information Science in Engineering, 17(3), 031013 (2017)

3. Huang, H. M., Rauch, U., Liaw, S. S.: Investigating learners' attitudes toward virtual reality learning environments: Based on a constructivist approach. Computers \& Education 55(3), 1171-1182 (2010)

4. Sanchez-Vives, M. V.,Slater, M.: From presence to consciousness through virtual reality. Nature Reviews Neuroscience, 6(4), 1-34 (2005)

5. Muhanna, M. A.: Virtual reality and the CAVE: Taxonomy, interaction challenges and research directions. Journal of King Saud University-Computer and Information Sciences, 27(3), 344-361 (2015)

6. Diemer, J., Alpers, G. W., Peperkorn, H. M., Shiban, Y., Mühlberger, A.:The impact of perception and presence on emotional reactions: a review of research in virtual reality. Frontiers in psychology, 6 (26), 1-9 (2015)

7. Christofi, M., Kyrlitsias, C., Michael-Grigoriou, D., Anastasiadou, Z., Michaelidou, M., Papamichael, I., Pieri, K.: A tour in the archaeological site of choirokoitia using virtual reality: a learning performance and interest generation assessment. In Advances in Digital Cultural Heritage, pp. 208-217. Springer, Cham (2018)

8. Behr, J., Fröhlich, T., Knöpfle, C., Kresse, W., Lutz, B., Reiners, D., Schöffel,F., Ehr, J. : The Digital Cathedral of Siena--Innovative Concepts for Interactive and lmmersive Presentation of Cultural Heritage Sites. 57-71 (2001)

9. Kontogianni, G., Georgopoulos, A., Saraga, N., Alexandraki, E., Tsogka, K.: 3D virtual reconstruction of the Middle Stoa in the Athens Ancient Agora. ISPRS-International Archives of the Photogrammetry, Remote Sensing and Spatial Information Sciences, 40, 125131 (2013)

10. Guidi, G., Russo, M.: Diachronic 3D reconstruction for lost Cultural Heritage. International Archives of the Photogrammetry, Remote Sensing and Spatial Information Sciences, 38(W16), 371-376 (2011)

11. Loizides, F., El Kater, A., Terlikas, C., Lanitis, A., Michael, D.: Presenting cypriot cultural heritage in virtual reality: A user evaluation. In Euro-Mediterranean Conference, pp. 572579. Springer, Cham.(2014)

12. Andreou, A: Berengaria, The Hotel of the Kings. iWrite, Nicosia (2016)

13. Goradia, I., Doshi, J., Kurup, L.: A review paper on oculus rift \& project morpheus. International Journal of Current Engineering and Technology, 4(5), 3196-3200 (2014)

14. Babbie, E.: The Practice of Social Research. 13edn. Belmont, Wadsworth (2013)

15. Papanastasiou, K., Papanastasiou, E .: Educational Research Methodology. 1edn. Private, Nicosia (2005)

16. Barbat, A. H., Moya, F. Y., Canas.: Damage scenarios simulation for seismic risk assessment in urban zones. Earthquake spectra, 12(3), 371-394 (1996)

17. Baka, E., Stavroulia, K. E., Magnenat-Thalmann, N., Lanitis, A.: An EEG-based Evaluation for Comparing the Sense of Presence between Virtual and Physical Environments. In Proceedings of Computer Graphics International, 107-116 (2018) 